\title{
Adrenal Insufficiency Complicated with Antiphospholipid Syndrome (APS)
}

\author{
Naohito Fujishima, Atsushi Komatsuda, Hideaki Ohyagi, Masumi Fujishima, Mitsunori Tada, \\ Hiroshi Ohtani, Hideki Wakui, Makoto Hirokawa and Ken-ichi Sawada
}

\begin{abstract}
We describe a 69-year-old woman with bilateral adrenal hemorrhage complicated with antiphospholipid syndrome (APS). She was hospitalized with nausea and vomiting in September 2003. Laboratory data demonstrated hyponatremia, hypoglycemia and prolongation of activated partial thromboplastin time (aPTT). Abdominal computed tomography showed bilateral adrenal enlargement. In October 2003, she demonstrated altered mental status with progressive hyponatremia, a high level of ACTH, and a low level of serum cortisol. She also showed thrombocytopenia, anti-cardiolipin IgG antibody, anti- $\beta 2$ GPI antibody, and lupus anticoagulants. After four months, anti-cardiolipin IgG antibody was still positive. Based on these findings, she was diagnosed as having APS complicated with adrenal insufficiency due to hemorrhagic infarction. After treatment with corticosteroid, a low dose of aspirin and normal saline infusion, her condition quickly improved. Platelet counts and aPTT were also normalized. To our knowledge, this is the second Japanese case of APS complicated with bilateral adrenal hemorrhage. APS should be considered an important underlying cause of adrenal insufficiency.
\end{abstract}

Key words: adrenal hemorrhagic infarction, adrenal insufficiency, antiphospholipid syndrome

(DOI: 10.2169/internalmedicine.45.1603)

\section{Introduction}

Antiphospholipid syndrome (APS) is characterized by recurrent arterial and/or venous thrombosis, spontaneous abortion, and thrombocytopenia $(1,2)$. Spontaneous arterial and/ or venous thromboembolism may involve any part of the vasculature. Arterial and/or venous thromboembolism may also occur in any organs including the brain, lung, heart, and liver, however, adrenal infarction and hemorrhage due to APS are rare events (1-7). To our knowledge, there have been few reports of adrenal hemorrhage complicated with APS except for one recent case in Japan (8). We describe here a second Japanese patient with adrenal insufficiency caused by bilateral adrenal hemorrhage secondary to APS.

\section{Case Report}

On September 10, 2003, a 69-year-old woman consulted her family doctor because of recent onset of nausea and vomiting. She had been taking anti-hypertensive drugs for fifteen years. She did not have any history of thrombosis or spontaneous abortion, and had a daughter by typical vaginal delivery. Although she was given oral levofloxacin and received intravenous fluids, her condition worsened with the development of hyponatremia and hypoglycemia. Then she was transferred to a hospital for further examination. Chest $\mathrm{X}$-ray did not detect any obvious abnormality and ultrasonic cardiogram (UCG) showed normal left ventricular contraction (70\% ejection fraction) without pericardial effusion. Abdominal computed tomography (CT) showed enlargement of bilateral adrenal glands with especially increased density on the left side (Fig. 1A). Endoscopic examination of the gastro-intestinal tract did not detect any abnormality. On October 24 , she suddenly developed disturbed consciousness (JCS II-30) without any other neurological findings, and blood pressure was $136 / 90 \mathrm{mmHg}$. Laboratory examination demonstrated severe hyponatremia (serum sodium $113 \mathrm{mEq} /$ 1), normocytic anemia (hemoglobin $9.0 \mathrm{~g} / \mathrm{dl}$ ), thrombocytopenia (platelet count $6.5 \times 10^{4} / \mu \mathrm{l}$ ), and prolonged activated 


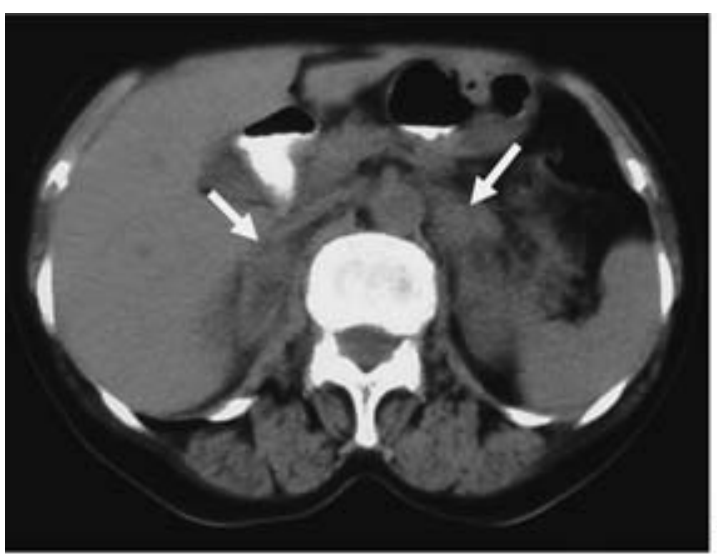

(A)

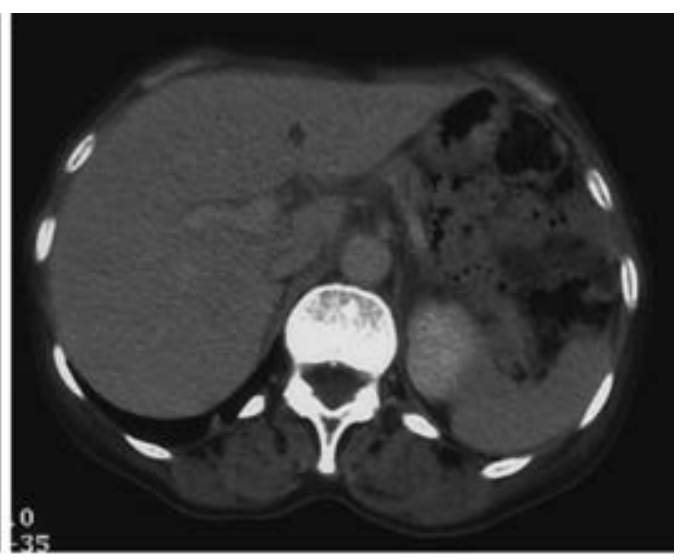

(B)

Figure 1. Abdominal computed tomography showed bilateral adrenal enlargement with an increased density at onset (arrows), in particular in the left adrenal gland between the abdominal aorta and the left kidney (A). After 3 months, the adrenal gland size was reduced and it showed less density (B).

partial thromboplastin time (aPTT) $(60.1 \mathrm{sec})$. At that time, she was treated with intravenous infusion of prednisolone (30 mg/day) and normal saline for three days, and her condition became almost normal. The cause of hyponatremia was not detected at that time, and she was then given $10 \mathrm{mg}$ oral prednisolone for three days, followed by the administration of $0.1 \mathrm{mg}$ oral fludrocortisone daily. The adrenocorticotropin (ACTH) level was markedly elevated (1390 pg/ml) and serum cortisol was $6.4 \mu \mathrm{g} / \mathrm{dl}$. Since she had progressive anemia (hemoglobin $7.3 \mathrm{~g} / \mathrm{dl}$ ) and persistent thrombocytopenia (platelet count $7.3 \times 10^{4} / \mu \mathrm{l}$ ), she was referred to our hospital on November 25.

On admission, her height was $146 \mathrm{~cm}$, and body weight $43.6 \mathrm{~kg}$. Her blood pressure was $142 / 80 \mathrm{mmHg}$ with a pulse rate of $80 / \mathrm{min}$. Her consciousness was alert. Conjunctivae were anemic, but not icteric. There was pigmentation on her face and finger. Her lungs and heart were normal. Pre-tibial and ankle edema was observed. There was neither erythema nor arthritis. Laboratory examination demonstrated normocytic anemia, thrombocytopenia, and prolonged aPTT. The hemoglobin level was $7.1 \mathrm{~g} / \mathrm{dl}$, red cell count 2.45 million/ $\mu \mathrm{l}$, hematocrit $21.9 \%$, reticulocytes $2.49 \%$, platelet count $10.7 \times 10^{4} / \mu \mathrm{l}$, and aPTT $67.0 \mathrm{sec}$. The white cell count was $4800 / \mu$ with $76 \%$ neutrophils, $18 \%$ lymphocytes, $4 \%$ monocytes, and $2 \%$ eosinophils. The aspartate aminotransferase level was $14 \mathrm{U} / \mathrm{l}$, alanine aminotransferase $5 \mathrm{U} / \mathrm{l}$, lactate dehydrogenase $198 \mathrm{U} / \mathrm{l}$, blood urea nitrogen $8.5 \mathrm{mg} / \mathrm{dl}$, creatinine $0.6 \mathrm{mg} / \mathrm{dl}$, total protein $6.6 \mathrm{~g} / \mathrm{dl}$, and albumin 3.8 $\mathrm{g} / \mathrm{dl}$. Serum sodium was $136 \mathrm{mEq} / \mathrm{l}$, potassium $2.9 \mathrm{mEq} / \mathrm{l}$, and chloride $99 \mathrm{mEq} / \mathrm{l}$. The level of serum ferrum was 28 $\mu \mathrm{g} / \mathrm{dl}$, ferritin $182.7 \mathrm{ng} / \mathrm{ml}$, unsaturated iron binding capacity (UIBC) $137 \mu \mathrm{g} / \mathrm{dl}$, and haptoglobin $141 \mathrm{mg} / \mathrm{dl}$. Direct and indirect Coombs test were negative. C-reactive protein was $0.3 \mathrm{mg} / \mathrm{dl}$. Endocrine examination demonstrated a decreased level of cortisol $(6.5 \mu \mathrm{g} / \mathrm{dl})$, dopamine $(<0.02 \mathrm{ng} / \mathrm{ml})$, and aldosterone $(<10 \mathrm{pg} / \mathrm{ml})$, and an elevated level of ACTH $(990$ $\mathrm{pg} / \mathrm{ml}$ ). The free T3 level was $1.6 \mathrm{pg} / \mathrm{ml}$, free T4 $1.2 \mathrm{ng} / \mathrm{dl}$, and TSH $3.60 \mu \mathrm{IU} / \mathrm{ml}$. On immunological examination, the patient was positive for anti-cardiolipin IgG antibody, anti- $\beta$ 2 glycoprotein I ( $\beta 2 \mathrm{GPI})$ antibody, and lupus anticoagulants, but negative for anti-cardiolipin IgM antibody, antinuclear antibodies (ANA), or anti-adrenal cortex antibodies. Bone marrow study showed normocellular marrow including megakaryopoiesis with normal karyotype. Urinalysis did not show proteinuria or hematuria. Chest X-ray showed cardiomegaly (cardiothoracic ratio $70.8 \%$ ) with mild bilateral pleural effusion. Electrocardiography demonstrated a low voltage due to pericardial effusion without ST-T change. At that time, UCG showed moderate pericardial fluid, but left ventricular movement maintained sufficient function with $70 \%$ ejection fraction, without dilatation of the inferior vena cava. An abdominal CT showed reduction of the bilateral adrenal enlargement with diminished attenuation values (Fig. 1B). Angiography of the adrenal glands did not demonstrate any evidence of thrombosis or non-staining lesion. On brain CT, there were no findings that would suggest pituitary tumor. Tuberculin reaction test was negative. Although there was pericardial effusion, thrombocytopenia, and positive ANA, this case did not satisfy the criteria for systemic lupus erythematosus (9). With these findings, she was suspected of having adrenal insufficiency due to bilateral hemorrhagic infarction caused by primary APS. Due to worsening edema and pericardial effusion, which was probably a side effect of fludrocotisone being a strong mineralcorticoid, she was treated with $20 \mathrm{mg}$ of hydrocortisone and $81 \mathrm{mg}$ of aspirin daily. Thereafter, her edema and pericardial effusion disappeared, and laboratory findings were normalized without any new episodes of thrombosis. Since immunological re-examination on March 2004 was positive for anti-cardiolipin IgG antibody, but negative for lupus anticoagulants or anti-ß32GPI antibody, she was finally diagnosed as having primary APS. Immunological findings had nor- 
malized by March 2005, with a white cell count $5900 / \mu \mathrm{l}$, hemoglobin $12.8 \mathrm{~g} / \mathrm{dl}$, platelet count $25.9 \times 10^{4} / \mu \mathrm{l}$, aPTT $30.6 \mathrm{sec}$, serum sodium $144 \mathrm{mEq} / \mathrm{l}$, potassium $4.2 \mathrm{mEq} / \mathrm{l}$, chloride $99 \mathrm{mEq} / \mathrm{l}$, and ACTH $29 \mathrm{pg} / \mathrm{ml}$.

\section{Discussion}

Primary adrenal insufficiency is caused by autoimmune diseases, infection, metastatic cancer, adrenal hemorrhage and/or infarction. Tuberculous adrenalitis was previously the most common cause of primary adrenal insufficiency. However, at present, the most frequent cause in Western countries is destruction of the adrenal cortex by an autoimmune process $(3,10)$.

Acute adrenal insufficiency complicated by adrenal hemorrhage is rare but it is life threatening (11). Postmortem diagnosis was commonly made before CT scan became widely available (12). The advent of CT scan has demonstrated adrenal hemorrhage as a more frequent cause of adrenal insufficiency than that had been recognized previously. The cause of adrenal hemorrhage and/or infarction is well known to be associated with meningococcemia (WaterhouseFriderichsen syndrome) or pseudomonas aeruginosa infection.

Since APS was recognized in 1986, APS had been reported as a cause of adrenal hemorrhage and/or infarction (3-7, 13). In 2003, Espinosa et al reviewed the clinical characteristics of 86 patients with adrenal involvement secondary to APS, and they suggested that the close relationship between APS and hypoadrenalism was more than coincidental $(3,6)$. Their study demonstrated primary APS in $71 \%$ of cases showing adrenal involvement as a complication of APS, and adrenal insufficiency was the first clinical manifestation in more than one-third of those cases $(3,6)$. Interestingly, the present lesion was limited to the adrenal glands and catastrophic APS was ruled out based on this finding, although most of their reported cases were based on catastrophic APS with multiple organ involvement (3-6).

The pathogenesis of adrenal vein thrombosis due to APS can be explained in part by the unique nature of the vascular anatomy of the adrenal glands, which have a rich arterial supply but a limited venous return via a single vein. This anatomical fragility is likely to be vulnerable to the formation of platelet thrombi. Adrenal vein thrombosis may lead to edema of the adrenal gland itself, and secondary obstruction of the peculiar arterial supply results in hemorrhagic infarction (3). The most common setting is a stressed patient receiving long-term anticoagulants, such as warfarin and aspirin, for the prevention of pulmonary and cardiac embolism or other thromboembolism.

Takebayashi et al reported the first Japanese case of APS with adrenal failure after surgery for myoma uteri (8). Although a fresh hemorrhagic lesion is generally demonstrated as a high density area on CT imaging (14), adrenal hemorrhage has been visualized as a normal density lesion in some cases including the case reported by Takebayashi et al (8). In the present case, serial abdominal CT showed enlargement of the adrenal glands with a slightly increased density, then CT obtained three months later showed diminution of the gland size with a diminished density. These changes on CT imaging could be explained by the process of healing after adrenal hemorrhage as previously reported $(7,15)$. Accordingly, we considered that adrenal insufficiency was caused by bilateral adrenal hemorrhagic infarction and edema following adrenal venous thrombosis.

There was no obvious prior history of other thrombosis or spontaneous abortion in this case. With this suspicious thrombotic episode and laboratory data demonstrating thrombocytopenia, anemia, prolonged aPTT, and the presence of anti-cardiolipin antibodies and anti- $\beta 2$ GPI antibodies, we diagnosed this patient as having primary APS (3).

Anticoagulation therapy including warfarin, anti-platelet drugs, and/or heparin are recommended in addition to hormone replacement therapy for adrenal insufficiency complicated by APS (3). Espinosa et al reported the efficacy of long-term anticoagulation therapy in preventing new thrombosis in APS with adrenal complication $(3,6)$. Since our case was not complicated with other obvious organ involvement, we have been treating our patient with a low dose of aspirin. Since initiation of this treatment, the patient has not developed any new episodes of thrombosis. Although the anti-cardiolipin $\operatorname{IgG}$ was still detectable four months later, this immunological abnormality spontaneously disappeared one year later under treatment with only hydrocortisone and aspirin.

In conclusion, adrenal insufficiency with APS is relatively rare, but it may be more frequent than previously recognized. When we encounter patients with adrenal insufficiency, we should investigate other thrombotic episodes, past history of spontaneous abortion, and the value of platelets and/or aPTT. If there are suspicious findings, they should be recorded in detail in order to evaluate the possibility of APS.

\section{References}

1. Chi HS. Recent advances in the diagnosis of antiphospholipid syndrome. Int J Hematol 76 Suppl 2: 47-51, 2002.

2. Wilson WA, Gharavi AE, Koike T, et al. International consensus statement on preliminary classification criteria for definite antiphospholipid syndrome: report of an international workshop. Arthritis Rheum 42: 1309-1311, 1999.

3. Espinosa G, Cervera R, Font J, Asherson RA. Adrenal involve- ment in the antiphospholipid syndrome. Lupus 12: 569-572, 2003. 4. Asherson RA, Cervera R, Piette JC, et al. Catastrophic antiphospholipid syndrome: clues to the pathogenesis from a series of 80 patients. Medicine (Baltimore) 80: 355-377, 2001.

5. Asherson RA, Cervera R, Piette JC, et al. Catastrophic antiphospholipid syndrome. Clinical and laboratory features of 50 patients. Medicine (Baltimore) 77: 195-207, 1998. 
6. Espinosa G, Santos E, Cervera R, et al. Adrenal involvement in the antiphospholipid syndrome: clinical and immunologic characteristics of 86 patients. Medicine (Baltimore) 82: 106-118, 2003.

7. Tauchmanova L, Rossi R, Coppola A, et al. Antiphospholipid syndrome, adrenal failure, dilated cardiomyopathy and chronic hepatitis: an unusual manifestation of multiorgan autoimmune injury? Eur J Endocrinol 139: 641-645, 1998.

8. Takebayashi K, Aso Y, Tayama K, Takemura Y, Inukai T. Primary antiphospholipid syndrome associated with acute adrenal failure. Am J Med Sci 325: 41-44, 2003.

9. Hochberg MC. Updating the American College of Rheumatology revised criteria for the classification of systemic lupus erythematous. Arthritis Rheum 40: 1725, 1997.

10. Oelkers W. Adrenal insufficiency. N Engl J Med 335: 1206-1212, 1996.
11. Kawashima A, Sandler CM, Ernst RD, et al. Imaging of nontraumatic hemorrhage of the adrenal gland. Radiographics 19: 949963, 1999.

12. Caron $\mathrm{P}$, Chabannier MH, Cambus JP, Fortenfant F, Otal P, Suc JM. Definitive adrenal insufficiency due to bilateral adrenal hemorrhage and primary antiphospholipid syndrome. J Clin Endocrinol Metab 83: 1437-1439, 1998.

13. Asherson RA, Hughes GR. Hypoadrenalism, Addison's disease and antiphospholipid antibodies. J Rheumatol 18: 1-3, 1991.

14. Dunnick NR. Hanson lecture. Adrenal imaging: current status. AJR 154: 927-936, 1990.

15. Wolverson MK, Kannegiesser H. CT of bilateral adrenal hemorrhage with acute adrenal insufficiency in the adult. AJR 142: 311314, 1984.

(C) 2006 The Japanese Society of Internal Medicine

http://www.naika.or.jp/imindex.html 